\title{
Increased Reporting of Sexual Minority Orientation from 2009 to 2017 in England and Implications for Measuring Sexual Minority Health Disparities
}

\author{
Sarah MacCarthy, ScD, ${ }^{1}$ Catherine L. Saunders, $\mathrm{PhD},{ }^{2}$ and Marc N. Elliott, $\mathrm{PhD}^{1}$
}

\begin{abstract}
Purpose: Sexual minority adults experience considerable mental and physical health disparities compared to their heterosexual counterparts, but changes in these disparities over time may, in part, reflect changes in how the same people self-identify their sexual orientation. Therefore, we estimated between-cohort and within-person changes in self-identified sexual orientation to better understand changes in sexual orientation-based health disparities over the life course.

Methods: We examined 2009 to 2015 changes among 5712 adults 19-25 years of age from the Longitudinal Study of Young People in England (LSYPE) to validate a synthetic cohort approach applied to cross-sectional data on 1,168,346 adults 18-74 years of age $(n=29,677$ sexual minority adults) from the General Practice Patient Survey (GPPS), estimating 2009 to 2017 changes in sexual orientation identification.

Results: Heterosexual identification for adults 18-24 years of age fell three percentage points from 2009 to 2017. Approximately half of those 26-32 years of age, who identified as sexual minority adults in 2017, may not have identified as such 8 years earlier. LSYPE and GPPS results were similar, validating the synthetic cohort application. Within-cohort analyses estimated that $2.1 \%$ and $2.7 \%$ of heterosexual-identified women and men 18-24 years of age in 2009, respectively, changed identification by 2017. Smaller changes occurred for adults 25-34 years of age (1.0\% and $1.2 \%$, respectively), with still smaller differences at older ages.

Conclusion: Changes in health disparities may reflect people newly identifying as a sexual minority as well as longitudinal changes in those long identifying as sexual minority adults. Future research should measure the health of both early- and late-identifying sexual minority adults, as their health care and policy needs may differ.
\end{abstract}

Keywords: General Patient Practice Survey, health disparities, longitudinal data, Longitudinal Study of Young People in England, sexual orientation, synthetic cohort

\section{Introduction}

G LOBALly ${ }^{1}$ AND IN THE UNITED KingDom, ${ }^{2,3}$ health disparities are evident for sexual minority adults, including lesbian, gay, bisexual, or "other" adults who self-identify with a sexual minority sexual orientation; they experience high rates of physical and mental health concerns and limited access to services. ${ }^{1-3}$ In the United Kingdom, sexual minority adults and groups such as men reporting sex with men (MSM) face higher odds of adverse mental, physical, and sexual health outcomes. ${ }^{2,3}$ Recent policies and programs have called for specific action to reduce these disparities. ${ }^{4,5}$ The evolving sociopolitical environment may influence sexual orientation-based disparities in two ways. First, antidiscrimination measures can improve health care access; a reduction in disparities would reflect improvements for a constant cohort of sexual minority adults. Second, the shifting environment could lead to more people self-identifying as sexual minority adults, changing the populations involved in calculating sexual orientation-based health disparities.

Estimated changes in sexual orientation-based health disparities combine (a) changes in health among those who identified as sexual minority adults at baseline and followup, relative to those not identifying as sexual minority adults at either wave, (b) differences in health at baseline between those not identifying as sexual minority adults at either wave and those identifying as a sexual minority adult only at follow-up, and (c) differences in health at follow-up between sexual minority adults who did and did not originally identify

\footnotetext{
${ }^{1}$ RAND Corporation, Santa Monica, California, USA.

${ }^{2}$ Primary Care Unit, Department of Public Health \& Primary Care, University of Cambridge, Cambridge, United Kingdom.
} 
as sexual minority adults. As many health disparities are based on characteristics that change little, component " $a$ ", is usually assumed to be the only important factor when examining changes in disparities. Here, component "b" is likely to be relatively minor, given the small size of those changing relative to the size of the heterosexual group. Component "c" may be consequential if a substantial proportion of sexual minority adults at follow-up did not report a sexual minority identity at baseline. Little is known about the relative contributions of these components to changes in disparities, but the answer promises to help efforts to reduce these disparities in the United Kingdom and elsewhere. If there is substantial change in identification within a cohort, progress in reducing health disparities may be greatly underestimated or overestimated.

The limited number of large datasets with sexual orientation questions inhibits examination of how sexual orientation identification changes over time.$^{6,7}$ England is one of the few countries that collects large-scale nationally representative data on sexual orientation, including the Longitudinal Study of Young People in England (LSYPE) and the General Practice Patient Survey (GPPS). We validated a synthetic cohort technique-which uses repeated cross-sectional data in the same population to approximate what might be learned from longitudinal data ${ }^{8}$ - to estimate within- and betweencohort changes in sexual orientation in the GPPS data, to understand how sexual orientation reporting changed from 2009 to 2017 and the implications for measuring changes in sexual minority health disparities.

\section{Methods}

Our primary goal was to estimate changes in selfidentified sexual orientation over time among different age cohorts to inform the interpretation of observed changes in health disparities affecting sexual minority adults. Ideally, one would track a constant cohort over time; in the absence of longitudinal data suitable for all age cohorts, we applied a synthetic cohort approach to repeated cross-sectional data to estimate these changes for all age cohorts and used longitudinal data available only for young adults to validate the synthetic cohort approach where possible.

\section{Data}

The LSYPE is a nationally representative longitudinal cohort study following young people in England born during 1989-1990, with weights accounting for selection probabilities. ${ }^{9}$ Almost all children in England were eligible to be included. The few who were excluded were children not on a school roll and children residing in the United Kingdom for education purposes only. We used data $(n=5712)$ from sweeps 6 to 8 collected during 2009-2010 and in 2015 (Supplementary Table S1). LSYPE data were accessed from the U.K. Data Archive ${ }^{10}$ through Secure Lab (approved for this use by project 128738).* Ethical approval for the

*The data used in this analysis are classified as "Controlled Data" and before access was granted to the research team, the researcher working with the data (C.L.S.) was formally accredited and an application was made for the data usage for this specific study, and this application was approved by the relevant Data Access Committee, and by the data depositor, University College London, UCL Institute of Education, Centre for Longitudinal Studies.
LSYPE study itself was secured from the National Health Service Research Ethics Committee (REC Reference 14/LO/0096). To prevent statistical disclosure of small groups for LSYPE, unadjusted estimates were not permitted; instead, weighted and covariate-adjusted predictions, adjusting for month of birth, from multivariable multinomial logistic models were used. English schools were the primary sampling units, selected using probability proportional to size sampling; pupils were selected within schools with probabilities dependent on ethnic groups.

The GPPS is an annual cross-sectional National Health Service (NHS) England-sponsored survey of patient experience in primary care (Supplementary Table S2), mailed to 2.7 million patients in England selected through stratified random sampling among those continuously registered with a general practice for at least 6 months (General Practice registration in the United Kingdom has almost universal coverage $)^{11}$ and who have not received a copy of the survey in the past year. ${ }^{12-14}$ We used data from April to June 2009 $(n=449,815)$ and January to March $2017(n=718,531)$; GPPS data were shared with the University of Cambridge for this use under a Data Sharing Agreement with NHS England. ${ }^{15,16}$ Data on population changes in the size, age, and sex structure of England came from the Office for National Statistics (ONS) ${ }^{17}$ census and other population data sources. All analyses were carried out at the University of Cambridge.

\section{Sexual orientation}

GPPS and LSYPE participants were asked, "Which of the following best describes how you think of yourself?" Both included response options of "Heterosexual/Straight," "Gay/Lesbian,"' "Bisexual," and "Other'” we excluded GPPS respondents who chose "Prefer not to say" because LSYPE did not include this option.

\section{Age}

We used respondent birth year and month in LSYPE analyses to calculate age. In GPPS, respondents were asked, "How old are you?" with options "18-24," "25-34," and in 10-year age groups to "85 and over." We excluded GPPS respondents 75 years and older because of suppression of detailed data for older respondents needed for calibration to ONS data.

\section{Analyses}

All analyses were stratified by sex. Analysis 1 used LSYPE data to directly measure within-person changes in sexual orientation responses among younger adults, as the same people were asked their sexual orientation at two time points (Table 1). This gold-standard data allowed us to validate our proposed synthetic cohort method in cross-sectional GPPS data. To validate the synthetic cohort approach, Analysis 2 compared directly estimated LSYPE estimates of net within-cohort changes in sexual orientation responses to a synthetic cohort estimate from crosssectional GPPS data. Analysis 3 extended the GPPS synthetic cohort estimates of within-cohort changes to people 18-74 years of age; Analysis 4 calculated between-cohort sexual orientation changes. 
Table 1. Within-Person Transitions in Sexual Orientation Responses from 2009 to 2015 With Longitudinal Study of Young People in England Data

\begin{tabular}{lccc}
\hline $\begin{array}{l}\text { Of young people reporting this sexual } \\
\text { orientation at age 19 in 2009 }\end{array}$ & $\begin{array}{c}\text { The estimated \% reporting this sexual } \\
\text { orientation at age 25 in 2015 }\end{array}$ & $\begin{array}{c}\text { Women adjusted \% } \\
(95 \% \text { CI })\end{array}$ & $\begin{array}{c}\text { Men adjusted } \% \\
(95 \% \text { CI })\end{array}$ \\
\hline Heterosexual & Heterosexual & $96.7(96.1-97.4)$ & $96.7(95.9-97.5)$ \\
& Gay/Lesbian & $0.6(0.3-0.8)$ & $1.0(0.6-1.4)$ \\
& Bisexual & $2.0(1.4-2.5)$ & $1.4(0.9-2.0)$ \\
& Other & $0.8(0.4-1.1)$ & $0.9(0.5-1.3)$ \\
Gay/Lesbian & Heterosexual & $8.5\left(0^{\mathrm{b}}-19.9\right)$ & $2.4\left(0^{\mathrm{b}}-7.1\right)$ \\
& Gay/Lesbian & $55.8(34.6-76.9)$ & $95.0\left(88.2-100^{\mathrm{b}}\right)$ \\
& Bisexual & $26.3(7.8-44.8)$ & $2.6\left(0^{\mathrm{b}}-7.7\right)$ \\
Bisexual & Other & $9.4\left(0^{\mathrm{b}}-21.9\right)$ & $0.0(-)$ \\
& Heterosexual & $39.4(28.9-49.8)$ & $26.2(9.1-43.4)$ \\
& Gay/Lesbian & $11.0(4.2-17.8)$ & $34.9(15.9-53.8)$ \\
Other $^{\mathrm{a}}$ & Bisexual & $47.2(36.4-58.0)$ & $38.9(18.9-58.9)$ \\
& Other & $2.4\left(0^{\mathrm{b}}-5.7\right)$ & $0.0(-)$ \\
& Heterosexual & $50.6(22.5-78.8)$ & $50.3(0-100)^{\mathrm{b}}$ \\
& Gay/Lesbian & $0.0(-))$ & $49.7(0-100)^{\mathrm{b}}$
\end{tabular}

aChanges from "other" sexual orientation at age 19 were poorly estimated, with large CIs.

${ }^{\mathrm{b}}$ We estimated $95 \%$ CIs for these adjusted percentages using the Delta method based on an approximation to the normal distribution; where this resulted in estimates of $95 \%$ CIs $<0$ or $>100$, we truncated these estimates to 0 and 100 , respectively.

CI, confidence interval.

Analysis 2 (Table 2) compared directly estimated cohort changes in reported sexual orientation between ages 19 (in 2009) and 25 (in 2015) in LSYPE data to indirectly estimated GPPS changes among people from synthetic cohorts with mean ages of 21.0 and 28.7. While the LSYPE data directly estimated change over 6 years from age 19 starting in 2009 , the GPPS approach estimated changes over 7.75 years from age 21.0, also starting in 2009.

The GPPS approach created a synthetic cohort intended to represent those who were 18-24 years of age during AprilJune 2009 , but 7.75 years later, through a weighted average of 2017 responses of those 25-34 years of age in JanuaryMarch 2017 and those 18-24 years of age in 2017, with weights selected to create a mean age difference from the 2009 GPPS cohort to match the passage of 7.75 years (Supplementary Table S3). There are two differences between the LSYPE and GPPS estimates: (a) we examined 6 years of LSYPE change rather than 7.75 years of GPPS change, which should result in more change in GPPS than LSYPE; (b) we started at a mean age of 19 in LSYPE data rather than age 21 in GPPS data, which might result in less change in GPPS than LSYPE. Taken together, these factors may partially offset each other.

More generally, the synthetic cohort approach estimated the January to March 2017 counterpart of April to June 2009 GPPS respondents for a 2009 age group as a weighted average of the same age group in 2017 and the next-oldest age group (using GPPS age categories). The relative weights of the two 2017 GPPS groups incorporate existing GPPS weights, which account for sampling and nonresponse, and ONS population distributions by sex and 1-year age bands to account for mortality and other effects on the mean age of the 2009 GPPS group 7.75 years later (Supplementary Table S3).

Analysis 3 extended the synthetic cohort GPPS estimates of within-cohort changes from 2009 to 2017 for people
18-74 years of age in 2009 (Figs. 1 and 2 and Supplementary Table S4). Analysis 4 calculated the between-cohort changes between 2009 and 2017, that is, the differences overall and by age group in responses between the two time points (Table 3 and Supplementary Table S5). By comparing changes in self-identification by age group from Analysis 3 to these base rates, we estimated the proportion of 2017 sexual minority adults in a given age group, who would have identified as sexual minority adults 8 years before, partitioning each group into recently and less recently self-identifying as a sexual minority adult.

\section{Results}

Analysis 1: within-person transitions in sexual orientation responses (LSYPE)

We examined the same people's identification at two time points to illustrate how young people change their sexual orientation identification over time (Table 1). Of women and men who reported "heterosexual" at age 19, $96.7 \%$ also did so at age 25 . Changes from "heterosexual" were to "bisexual" (2.0\% of women and $1.4 \%$ of men) and to "gay/lesbian" ( $0.6 \%$ of women and $1.0 \%$ of men). Also, $55.8 \%$ of women and $95.0 \%$ of men who reported "gay/lesbian" at age 19 responded similarly at age 25 . Notably, $26.3 \%$ of women, but only $2.6 \%$ of men who reported "gay/lesbian" at age 19 reported "bisexual" at age 25 , whereas $8.5 \%$ of women and $2.4 \%$ of men who reported "gay/lesbian" at age 19 instead reported "heterosexual" at age 25. Changes were common for those reporting "bisexual" at age 19, with only $47.2 \%$ of women and $38.9 \%$ of men maintaining that response at age 25 . Women reporting a bisexual identity at age 19 were much more likely to report "heterosexual" than "gay/lesbian" at age 25 (39.4\% and $11.0 \%$, respectively), whereas the reverse was true for men $(34.9 \%$ reporting "gay" and 26.2\% "heterosexual" at age 25$)$. 


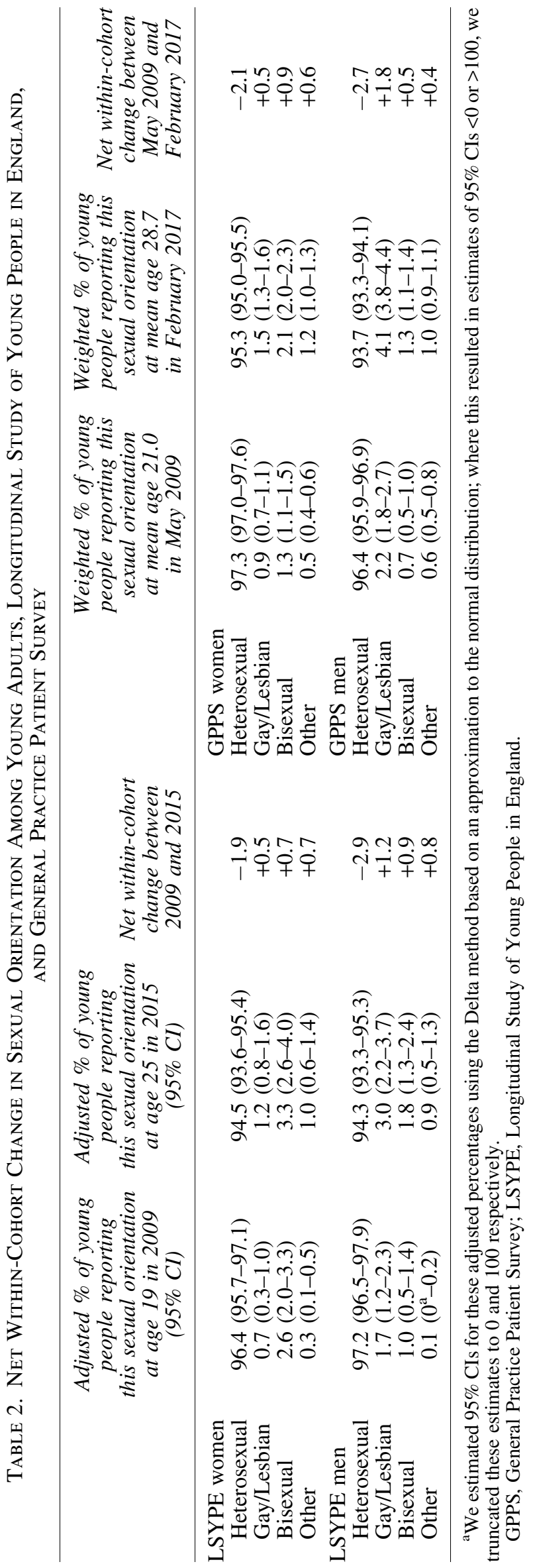

\section{Analysis 2: comparing LSYPE and GPPS net within-cohort changes in sexual orientation responses}

We conducted a validation exercise to compare indirectly estimated changes among GPPS respondents from mean age 21 to mean age 29 to gold standard directly estimated longitudinal changes in LSYPE between ages 19 and 25. The left side of Table 2 reports the LSYPE distribution of sexual orientation for a single cohort at age 19 in 2009 and age 25 in 2015; the right side displays the corresponding GPPS synthetic cohort estimates of change from mean age 21.0 in May 2009 to mean age 28.7 in February 2017. GPPS-estimated decreases in heterosexual orientation for young adults were very similar for LSYPE and GPPS (women: $-1.9 \mathrm{pp}$ LSYPE and -2.1pp GPPS; men: $-2.9 \mathrm{pp}$ LSYPE and -2.7pp GPPS). Similarly, the estimated increases across the three sexual minority categories differed minimally for women and somewhat more for men. LSYPE and GPPS results were sufficiently similar to validate the synthetic cohort approach in this setting, which we then applied to estimate within- and between-cohort changes across a broader range of adult ages.

\section{Analysis 3: estimates of within-cohort changes for all ages (GPPS)}

Among women 18-24 years of age in 2009 (Fig. 1 and Supplementary Table S4), there was an estimated withincohort net change of $-2.1 \mathrm{pp}$ for "heterosexual" by 2017 , and a 1.0pp decrease among women who were 25-34 years of age in 2009. Among men ages 18-24 and 25-34 in 2009 (Fig. 2 and Supplementary Table S4), "heterosexual", fell 2.7pp and 1.2pp, respectively, by 2017. In each instance, all three sexual minority categories increased.

If these within-cohort net changes only involved changes in the majority direction, we could estimate that, for example, $2.7 \% / 96.4=2.8 \%$ of men 18-24 years of age and identifying as heterosexual in 2009 no longer identified as heterosexual in 2017 (Table 2 and Supplementary Table S4). In reality, there were also changes in the less common direction (e.g., men identifying as bisexual in 2009 and then identifying as heterosexual in 2015; Table 1), so slightly more than $2.8 \%$ of men identifying as heterosexual in 2009 would have had to change to sexual minority identification by 2017 to produce the observed net withincohort change. The smaller size of the sexual minority categories makes the net change in the heterosexual category a close approximation of the proportion of the change from heterosexual to sexual minority categories.

\section{Analysis 4: between-cohort changes (GPPS)}

Table 3 (95\% confidence intervals in Supplementary Tables S5 and S6) shows the total 2009 to 2017 change in sexual orientation and estimates are reported in the next sections, except where noted. Total change in sexual orientation involves three components: change in sexual orientation within the same people; entrance of new sexual minority cohorts aging toward adulthood; and mortality of existing sexual minority cohorts. We found a nearly $1 \mathrm{pp}$ decrease in both women and men identifying as heterosexual, with statistically significant increases in each sexual minority 


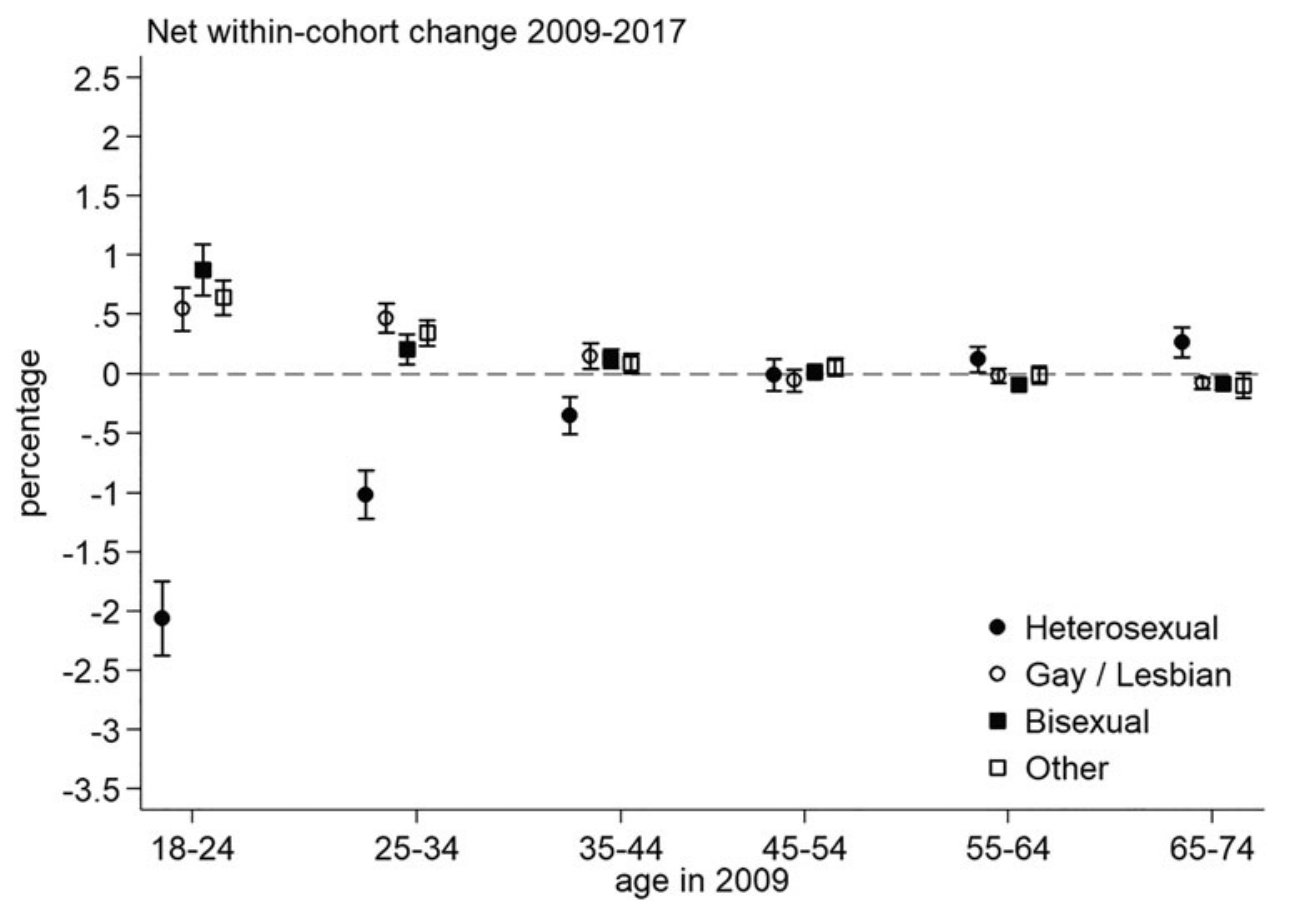

FIG. 1. Estimated net within-cohort changes from 2009 (April to June) to 2017 (January to March) in sexual orientation responses for women (General Practice Patient Survey). Notes: whisker bars are $95 \%$ confidence interval.

category. These are proportionately large increases $(+25 \%-$ $83 \%$ ) to each of the "gay/lesbian" and "bisexual" groups; "other" increased by $<15 \%$

In 8 years, heterosexual identification for ages 18-24 fell three percentage points (3.7pp among women and $2.3 \mathrm{pp}$ for men). The biggest increase for women was for "bisexual," increasing $2.5 \mathrm{pp}$ and nearly tripling in 8 years. "Lesbian" nearly doubled $(0.9 \%-1.6 \%)$. For men, the biggest increase was also for "bisexual," which more than doubled from $0.7 \%$ to $1.9 \%$, while "gay" increased from $2.2 \%$ to $3.1 \%$.

In $2017,6.4 \%$ of women and $5.9 \%$ of men $18-24$ years of age identified as sexual minority adults. For women, that per- centage $(6.4 \%)$ is double the all-ages sexual minority percentage in 2017 and triple the all-ages sexual minority percentage in 2009. Men 18-24 years of age in 2017 identified as sexual minority adults at 1.5 times the 2017 all-ages rate and at 1.8 times the 2009 all-ages rate.

In $2017,4.6 \%$ of women $25-34$ years of age identified as sexual minority adults compared to $2.4 \%$ in 2009 . Figure 1 and Supplementary Table S4 $(-2.1 \mathrm{pp}$ heterosexual for women 18-24 years of age in 2009 and 26-32 years of age in 2017) suggest that this 2.2pp difference largely reflects change in self-identified sexual orientation for the same people over time. By comparing the net increase to sexual

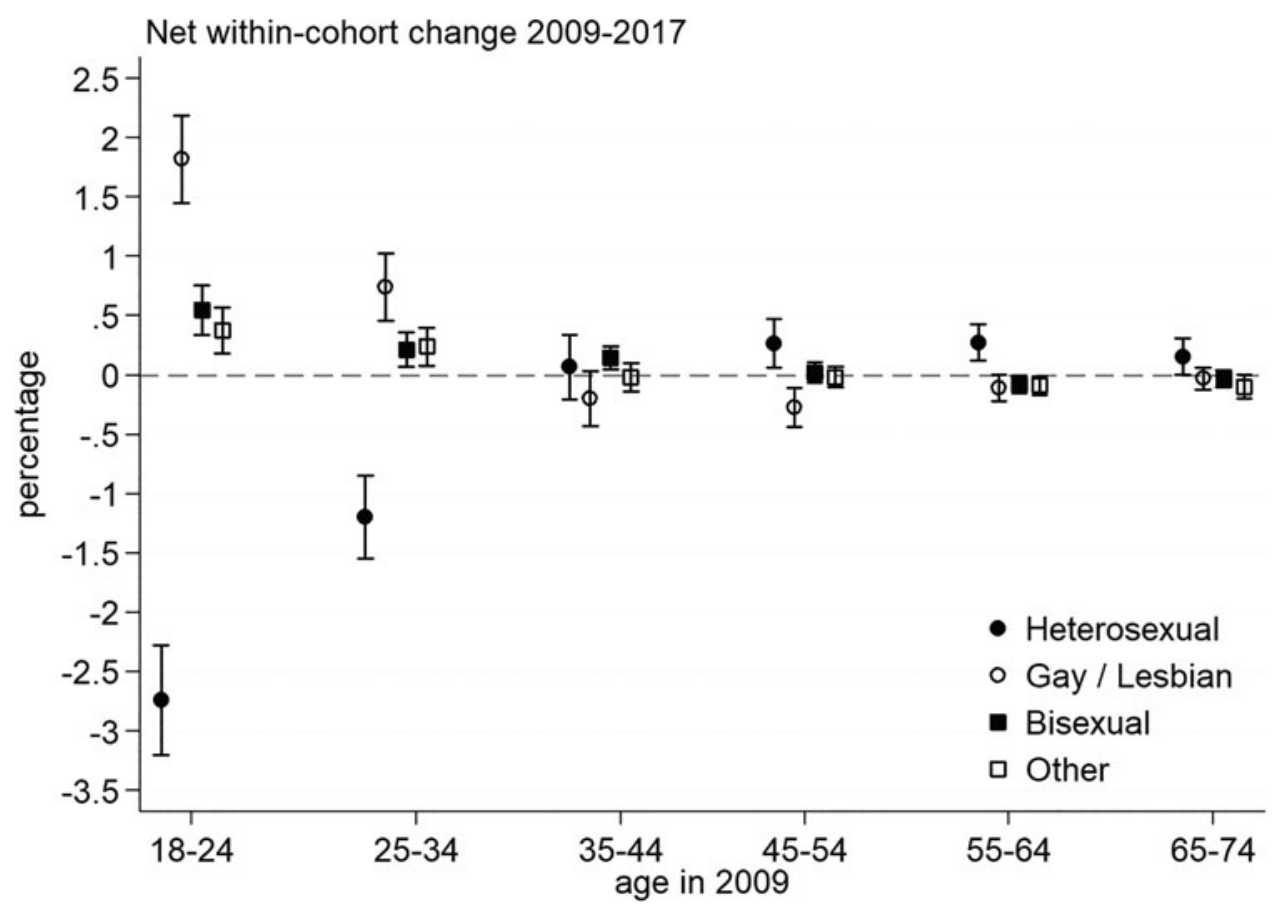

FIG. 2. Estimated net within-cohort changes from 2009 (April to June) to 2017 (January to March) in sexual orientation responses for men (General Practice Patient Survey). Notes: whisker bars are $95 \%$ confidence interval. 
Table 3. Estimated Between-Cohort Changes in Sexual Orientation 2009-2017 Using General Practice Patient Survey Data, Weighted Percentage, Stratified by Age

\begin{tabular}{|c|c|c|c|c|c|c|c|c|c|c|c|c|}
\hline & \multicolumn{3}{|c|}{ Heterosexual } & \multicolumn{3}{|c|}{ Gay/Lesbian } & \multicolumn{3}{|c|}{ Bisexual } & \multicolumn{3}{|c|}{ Other } \\
\hline & 2009 & 2017 & Difference & 2009 & 2017 & Difference & 2009 & 2017 & Difference & 2009 & 2017 & Difference \\
\hline \multicolumn{13}{|l|}{ Women } \\
\hline $18-24$ & 97.3 & 93.6 & -3.7 & 0.9 & 1.6 & 0.7 & 1.3 & 3.8 & 2.5 & 0.5 & 1.0 & 0.5 \\
\hline $25-34$ & 97.6 & 95.4 & -2.2 & 0.9 & 1.4 & 0.6 & 0.9 & 2.0 & 1.0 & 0.6 & 1.2 & 0.5 \\
\hline $35-44$ & 97.9 & 96.9 & -1.0 & 1.0 & 1.3 & 0.3 & 0.5 & 0.9 & 0.4 & 0.6 & 0.9 & 0.3 \\
\hline $45-54$ & 98.3 & 97.7 & -0.6 & 0.8 & 1.1 & 0.3 & 0.3 & 0.5 & 0.2 & 0.5 & 0.6 & 0.1 \\
\hline $55-64$ & 98.8 & 98.5 & -0.3 & 0.4 & 0.7 & 0.3 & 0.3 & 0.3 & 0.0 & 0.6 & 0.5 & 0.0 \\
\hline $65-74$ & 98.7 & 99.0 & 0.3 & 0.2 & 0.3 & 0.1 & 0.2 & 0.2 & -0.1 & 0.9 & 0.5 & -0.3 \\
\hline $75-84$ & 98.3 & 99.0 & 0.7 & 0.1 & 0.1 & 0.0 & 0.2 & 0.1 & -0.1 & 1.3 & 0.8 & -0.5 \\
\hline $85+$ & 97.8 & 98.6 & 0.8 & 0.2 & 0.1 & -0.2 & 0.5 & 0.2 & -0.3 & 1.5 & 1.1 & -0.4 \\
\hline All ages & 98.1 & 97.2 & -0.9 & 0.7 & 1.0 & 0.3 & 0.6 & 1.0 & 0.5 & 0.7 & 0.8 & 0.1 \\
\hline \multicolumn{13}{|l|}{ Men } \\
\hline $18-24$ & 96.4 & 94.1 & -2.3 & 2.2 & 3.1 & 0.8 & 0.7 & 1.9 & 1.2 & 0.6 & 0.9 & 0.3 \\
\hline $25-34$ & 95.7 & 93.7 & -2.0 & 2.8 & 4.2 & 1.3 & 0.7 & 1.2 & 0.5 & 0.9 & 1.0 & 0.1 \\
\hline $35-44$ & 95.6 & 94.7 & -0.9 & 3.1 & 3.4 & 0.3 & 0.5 & 0.8 & 0.3 & 0.8 & 1.1 & 0.3 \\
\hline $45-54$ & 96.8 & 95.9 & -0.9 & 2.1 & 2.8 & 0.7 & 0.5 & 0.6 & 0.0 & 0.6 & 0.7 & 0.1 \\
\hline $55-64$ & 97.9 & 97.4 & -0.5 & 1.1 & 1.5 & 0.4 & 0.5 & 0.6 & 0.1 & 0.5 & 0.5 & 0.0 \\
\hline $65-74$ & 98.3 & 98.3 & 0.0 & 0.6 & 0.8 & 0.2 & 0.4 & 0.4 & 0.0 & 0.7 & 0.4 & -0.3 \\
\hline $75-84$ & 98.1 & 98.5 & 0.4 & 0.5 & 0.5 & 0.0 & 0.3 & 0.3 & 0.0 & 1.0 & 0.6 & -0.3 \\
\hline $85+$ & 97.7 & 98.5 & 0.8 & 0.5 & 0.5 & 0.0 & 0.4 & 0.2 & -0.2 & 1.4 & 0.7 & -0.7 \\
\hline All ages & 96.7 & 96.0 & -0.8 & 2.0 & 2.5 & 0.5 & 0.5 & 0.8 & 0.3 & 0.7 & 0.8 & 0.1 \\
\hline
\end{tabular}

minority categories from Figure 1 and Supplementary Table S4 (the net decrease in heterosexual) within an age cohort from 2009 to 2017 to the total proportion identifying as sexual minority adults in that age cohort in 2017 , we estimated the minimum proportion of 2017 sexual minority adults who identified as heterosexual 8 years earlier (Supplementary Table S7). In 2017, almost half of sexual minority women (43.5\%) and men (43.4\%) 26-32 years old would not have identified as sexual minority adults 8 years earlier. The same is true for about one-fourth of those 33-42 years of age in 2017 (29.6\% of sexual minority women and $21.6 \%$ of men), and about one-seventh of sexual minority women 43-52 years of age in 2017 (14.6\%).

\section{Discussion}

We found substantial shifts from heterosexual to sexual minority identification. These changes differed by age and sex (more common at younger adult ages), consistent with literature on temporal variability in youth sexual minority identification. ${ }^{18,19}$ Such changes are likely associated with identity formation, ${ }^{18,19}$ as the transition to adulthood may coincide with sexual experimentation. ${ }^{20}$

We also found pronounced differences between younger and older age cohorts. ${ }^{14,21}$ Other research has emphasized "identity cohorts" based on the age at which people openly disclose their sexual orientation. ${ }^{22,23}$ Sex may also influence age cohort differences. Older lesbian women have reported recognizing same-sex attractions at an average age of 18 , beginning their first serious same-sex relationships 6.5 years later ${ }^{24}$; older gay men have reported earlier same-sex sexual contact, followed by a period of nonacceptance of self and then self-acceptance. ${ }^{25}$ Our results underscore the continued need to understand age- and identity-based cohort differences. These changes in self-identification are likely associ- ated with shifts in the social and political context around sexual minority rights; legal decisions have increased protections based on sexual orientation and parallel shifts in public opinion $^{26,27}$ signal the potential for continued increase in sexual minority self-identification.

To illustrate how lack of attention to changes in sexual orientation identification may limit our current understanding of sexual orientation-based health disparities, we developed two hypothetical scenarios (Supplementary Table S8 and Supplementary Data). Using EuroQual-5D, a measure of health-related quality of life (HRQOL) ${ }^{28}$ we showed that data suggest the HRQOL of sexual minority women 25-34 years of age at baseline declined faster compared with their heterosexual peers over 5.5 years if one ignored any change in self-reported sexual orientation. However, what is happening to constant cohorts of sexual minority women may be quite different from this apparent pattern; we illustrated two of several possible trajectories of health disparities that are consistent with both observed HRQOL data and this study's findings on changes in self-identified sexual orientation.

Our work underscores the value of more longitudinal health and sexual orientation data. Cross-sectional data include sexual minority adults who did and did not report sexual minority identity at younger adult ages. Our results suggest that both groups are common among sexual minority adults $26-42$ years of age. Changes in disparities are likely different for each group; for example, those who initially identified as heterosexual at 18-24 years of age might have better health and health care when they later identified as sexual minority adults than those who identified as a sexual minority adult earlier, if the former did not have the same history of discrimination. If so, their emergence into the sexual minority cohort would mimic a reduction in disparities, even if no change occurred within the cohort who identified 
as sexual minority adults from an early age. Alternatively, sexual minority adults who initially identified as heterosexual might have worse health and health care if they did not disclose relevant information to providers. If so, the reverse would be true.

Longitudinal research is needed to clarify progress in reducing disparities and to distinguish which respondents have which histories to better measure, understand, and address the health and health care experiences of both groups. A health campaign might use different images, eligibility screeners, and recommended actions for those who consistently identify as sexual minority adults versus those who do not. For example, different approaches to increasing HIV testing and linkage to care are now tailored to men who identify as gay and MSM who identified as heterosexual. ${ }^{2,29,30}$

\section{Limitations and strengths}

The synthetic cohort approach used age- and sex-stratified population estimates to weight each group and therefore only indirectly accounts for immigration, emigration, and mortality; such estimates should be interpreted as approximate. Second, there was some difference in the age groups used in the validation exercise (LYSPE: 19-25 vs. GPPS: 21-29) and slightly different sample frames (GPPS has near universal coverage of adults and LYSPE excludes a very small percentage of those who were in rare education settings), but our use of mean ages for the analysis limited potential differences in identity-related processes across samples. Nevertheless, the estimates for young adults were similar to direct longitudinal estimates. Unlike longitudinal data, synthetic-cohort data only allowed us to estimate net changes within a cohort; we could not uniquely estimate changes in opposing directions.

After removing the "Do not know or refuse" option for comparability, we found strikingly similar estimates for the proportion of women in England in 2017 who identified as sexual minority on the GPPS and the general household survey conducted by ONS ( $2.0 \%$ vs. $2.1 \%$, well within the ONS confidence intervals), but somewhat larger proportions of men who identified as sexual minority in GPPS (3.3\% vs. 2.6\%). ${ }^{31}$ Among other possibilities, men may have been more willing to identify as sexual minority in a survey where the results are used to measure health care for sexual minority adults than in a general household survey (ONS); our rates are also generally consistent with 2010-2012 Natsal-3 results for Britain. ${ }^{32}$ Nonetheless, these similarities support the external validity of our results and increase confidence in the inferences about the within-person changes among older adults that are unique to this study.

A strength of using GPPS is its large sample size over many years, which makes it possible to consider changes in older age groups. Information from both younger and older people in the same survey provides a second strength comparability. Despite some limitations, the nationally representative GPPS data provide one of the most comprehensive analyses of changes to sexual orientation selfidentification in the absence of longitudinal data.

\section{Conclusions}

We recommend assessing both within- and betweencohort differences between heterosexual and sexual minority men and women. Longitudinal cohort data are needed to more accurately determine whether changes in sexual orientationbased disparities reflect improvements for a constant cohort of sexual minority adults or a change in the populations identifying as sexual minority adults. Additional research is urgently needed to understand the demographic and health characteristics of both those who identify as sexual minority adults earlier and those who identify as such later in adulthood to help public health workers, providers, and policymakers reduce sexual orientation-based disparities.

\section{Acknowledgment}

The authors thank Biayna Darabidian for assistance with article preparation.

\section{Author Disclosure Statement}

No competing financial interests exist.

\section{Funding Information}

No funding was received for this article.

\section{Supplementary Material}

Supplementary Data

Supplementary Table S1

Supplementary Table S2

Supplementary Table S3

Supplementary Table S4

Supplementary Table S5

Supplementary Table S6

Supplementary Table S7

Supplementary Table S8

\section{References}

1. World Health Organization: Gender, Equity, \& Human Rights: FAQ on Health and Sexual Diversity: An Introduction to Key Concepts. Geneva, Switzerland: World Health Organization, 2016.

2. Mercer $\mathrm{CH}$, Prah P, Field N, et al.: The health and wellbeing of men who have sex with men (MSM) in Britain: Evidence from the third National Survey of Sexual Attitudes and Lifestyles (Natsal-3). BMC Public Health 2016;16:525.

3. Elliott MN, Kanouse DE, Burkhart Q, et al.: Sexual minorities in England have poorer health and worse health care experiences: A national survey. J Gen Intern Med 2015; 30:9-16.

4. Government Equalities Office: Equality Act 2010: Guidance. 2013. Available at https://www.gov.uk/guidance/ equality-act-2010-guidance Accessed June 3, 2019.

5. Government Equalities Office: LGBT Action Plan: Improving the Lives of Lesbian, Gay, Bisexual, and Transgender People. London, United Kingdom: Government Equalities Office, 2018.

6. Gates GJ: LGBT data collection amid social and demographic shifts of the US LGBT community. Am J Public Health 2017;107:1220-1222.

7. Grasso C, Goldhammer H, Funk D, et al.: Required sexual orientation and gender identity reporting by US health centers: First-year data. Am J Public Health 2019;109:11111118. 
8. Newell C: Methods and Models in Demography. New York, NY: The Guilford Press, 1988.

9. University College London, UCL Institute of Education, Centre for Longitudinal Studies: Next Steps: Sweeps 1-8, 2004-2016: Secure Access. [Data collection]. 4th ed. UK Data Service. SN: 7104. 2018. Available at http://doi.org/ 10.5255/UKDA-SN-7104-4 Accessed April 22, 2019.

10. University of Essex: UK Data Archive. Available at www .data-archive.ac.uk Accessed March 24, 2020.

11. Burch P, Doran T, Kontopantelis E: Regional variation and predictors of over-registration in English primary care in 2014: A spatial analysis. J Epidemiol Community Health 2018;72:532-538.

12. Campbell J, Smith P, Nissen S, et al.: The GP patient survey for use in primary care in the National Health Service in the UK-Development and psychometric characteristics. BMC Fam Pract 2009; 10:57.

13. Lyratzopoulos G, Elliott M, Barbiere JM, et al.: Understanding ethnic and other socio-demographic differences in patient experience of primary care: Evidence from the English General Practice Patient Survey. BMJ Qual Saf 2012;21:21-29.

14. Office for National Statistics: Sexual orientation, UK: 2017: Experimental statistics on sexual orientation in the UK in 2017 by region, sex, age, marital status, ethnicity and socioeconomic classification. 2019. Available at https://www.ons .gov.uk/peoplepopulationandcommunity/culturalidentity/ sexuality/bulletins/sexualidentityuk/2017 Accessed April 22, 2019.

15. NHS England: GP Patient Survey. Available at www.gppatient.co.uk/confidentiality Accessed March 24, 2020.

16. NHS England: GP Patient Survey. 2009-2017. Available at www.gp-patient.co.uk/confidentiality Accessed April 22, 2019.

17. Office for National Statistics: Dataset: Population estimates quinary age groups for UK constituent countries. 2015. Available at https://www.ons.gov.uk/peoplepopulationand community/populationandmigration/populationestimates/ datasets/populationestimatesquinaryagegroupsforukconsti tuentcountries Accessed May 2, 2019.

18. Ott MQ, Corliss HL, Wypij D, et al.: Stability and change in self-reported sexual orientation identity in young people: Application of mobility metrics. Arch Sex Behav 2011;40: 519-532.

19. Floyd FJ, Stein TS: Sexual orientation identity formation among gay, lesbian, and bisexual youths: Multiple patterns of milestone experiences. J Res Adolesc 2002;12:167-191.

20. Garofalo R, Wolf RC, Kessel S, et al.: The association between health risk behaviors and sexual orientation among a school-based sample of adolescents. Pediatrics 1998; 101:895-902.

21. Fredriksen Goldsen KI, Jen S, Muraco A: Iridescent life course: LGBTQ aging research and blueprint for the future-A systematic review. Gerontology 2019;65:253-274.
22. Dentato MP, Orwat J, Spira M, Walker B: Examining cohort differences and resilience among the aging LGBT community: Implications for education and practice among an expansively diverse population. J Hum Behav Soc Environ 2014;24:316-328.

23. Golombok S, Spencer A, Rutter M: Children in lesbian and single-parent households: Psychosexual and psychiatric appraisal. J Child Psychol Psychiatry 1983;24:551-572.

24. Averett P, Yoon I, Jenkins CL: Older lesbians: Experiences of aging, discrimination and resilience. J Women Aging 2011;23:216-232.

25. Neville S, Kushner B, Adams J: Coming out narratives of older gay men living in New Zealand. Australas J Ageing 2015;34(Suppl 2):29-33.

26. National Opinion Research Center (NORC) at the Unversity of Chicago: Same-sex marriage and gay rights: A shift in Americans' attitudes. 2015. Available at www.apnorc.org/ projects/Pages/HTML\%20Reports/same-sex-marriage-andgay-rights-a-shift-in-americans-attitudes0305-8272.aspx Accessed July 12, 2019.

27. Nat Cen Social Research: Charting changing attitudesSame-sex relationships. 2017. Available at www.natcen.ac .uk/blog/charting-changing-attitudes-\%E2\%80\%93-samesex-relationships Accessed June 3, 2019.

28. EuroQol Research Foundation: EQ-5D. Available at https:// euroqol.org/eq-5d-instruments Accessed May 21, 2020.

29. Prah P, Hickson F, Bonell C, et al.: Men who have sex with men in Great Britain: Comparing methods and estimates from probability and convenience sample surveys. Sex Transm Infect 2016;92:455-463.

30. Young RM, Meyer IH: The trouble with "MSM" and "WSW": Erasure of the sexual-minority person in public health discourse. Am J Public Health 2005;95:11441149.

31. Office for National Statistics: Sexual orientation, UK: 2017. 2019. Available at https://www.ons.gov.uk/peoplepopulation andcommunity/culturalidentity/sexuality/bulletins/sexual identityuk/2017 Accessed May 21, 2020.

32. Geary RS, Tanton C, Erens B, et al.: Sexual identity, attraction and behaviour in Britain: The implications of using different dimensions of sexual orientation to estimate the size of sexual minority populations and inform public health interventions. PLoS One 2018;13:e0189607.

Address correspondence to:

Marc N. Elliott, PhD

RAND Corporation

1776 Main Street

Santa Monica, CA 90401

USA

E-mail: elliott@rand.org 